Cahiers $d u$ MONDE RUSSE

\section{Cahiers du monde russe}

Russie - Empire russe - Union soviétique et États indépendants

$58 / 4 \mid 2017$

Varia

\title{
Olga Kucherenko, Soviet Street children and the Second World War: Welfare and Social Control under Stalin
}

\section{Dorena Caroli}

\section{(2) OpenEdition}

1 Journals

Édition électronique

URL : http://journals.openedition.org/monderusse/10159

DOI : 10.4000/monderusse.10159

ISSN : $1777-5388$

Éditeur

Éditions de l'EHESS

\section{Édition imprimée}

Date de publication : 1 octobre 2017

Pagination : 699-703

ISBN : 978-2-7132-2698-4

ISSN : $1252-6576$

\section{Référence électronique}

Dorena Caroli, «Olga Kucherenko, Soviet Street children and the Second World War: Welfare and Social Control under Stalin », Cahiers du monde russe [En ligne], 58/4 | 2017, mis en ligne le 01 octobre 2017, consulté le 06 janvier 2021. URL : http://journals.openedition.org/monderusse/10159; DOI : https://doi.org/10.4000/monderusse.10159 
Olga KUCHERENKO

Soviet Street children and the Second World War

Welfare and Social Control under Stalin

London - Oxford : Bloomsbury Academic, 2016, p. 245.

Ces vingt dernières années, l'ouverture des archives de l'Union soviétique a permis le renouvellement de l'histoire de l'enfance et de la jeunesse : dans ces archives, on découvre en effet de nouvelles informations sur les différentes périodes traversées par le pays, depuis la révolution d'Octobre jusqu'aux années cinquante. Grâce aux progrès de la recherche en histoire institutionnelle, en histoire sociale (histoire du contrôle de la société), en histoire culturelle et en anthropologie historique, un grand nombre de jeunes historiens (A. Ball, 1994 ; D. Caroli, 2004 ; A. Sal'nikova, 2007 ; J. Fürst, 2010) ont obtenu des résultats originaux permettant de mieux comprendre les réformes soviétiques dans leur dimension interdisciplinaire, de mieux cerner la relation entre État et société et l'essor des mécanismes totalitaires de gestion des problèmes sociaux. Ainsi, Olga Kucherenko, dans son premier livre Little Soldiers : 
How Soviet Children Went to War, 1941-45 (2011), a présenté des aspects inédits de la participation active de la jeunesse dans les actions de la Seconde Guerre mondiale : ce livre montrait le niveau de l'engagement des jeunes dans la défense héroïque du pays, qui s'inscrivait dans le système d'endoctrinement de la nouvelle génération par l'État soviétique de l'entre-deux-guerres.

Dans sa nouvelle recherche sur l'histoire de l'aide à l'enfance pendant la Seconde Guerre mondiale, cette jeune historienne d'origine russe s'appuie une fois encore sur un dépouillement impressionnant de documents des Archives d'État de la Fédération de Russie (GARF) et des Archives d'Histoire politique et sociale de l'État russe (RGASPI), ainsi que sur nombre d'études locales récentes sur la Seconde Guerre mondiale. Le thème de l'ouvrage est déjà connu (M.K. Stoole, 1988 ; R. Franco, 2006, A. Livschiz, 2007 ; N. Baron, 2017), mais l'auteur tente de reconstituer l'évolution des mesures de prise en charge [de welfare] mises en place par l'État pour faire face au problème des enfants orphelins de guerre, abandonnés dans les rues du pays, poussés par la faim dans la délinquance. Dans une certaine mesure, cette recherche confirme les hypothèses déjà formulées par les recherches précédentes sur l'assistance aux enfants abandonnés dans la Russie soviétique des années vingt. Le phénomène des enfants abandonnés de la période stalinenne - les besprizornye - n'était pas nouveau : l'Union soviétique l'avait déjà connu au cours des années vingt après les années révolutionnaires et durant la famine de 1921. Cependant les solutions choisies au cours des années vingt ne pouvaient servir de modèle pour le régime stalinien, en raison de la dérive totalitaire de l'approche adoptée vis-à-vis des problèmes sociaux, considérés sur le plan pénal d'un point de vue répressif dès $1935^{1}$.

Le livre est constitué d'une introduction et de deux parties, suivies d'un glossaire très utile pour comprendre le système complexe des institutions de prise en charge, d'annexes statistiques (sur les DPR [centres de premier accueil et de répartition], les colonies de travail et les colonies éducatives de travail pendant les années de guerre et parfois jusqu'en 1956), d'une bibliographie sélective et d'un index des noms. Dans son introduction, Kucherenko annonce que son point de départ a été l'étude de Julie de Graffenried, qui traite plutôt de l'exploitation des enfants appelés à la défense du pays : ils étaient placés à de nombreux postes de travail dans les usines et dans l'agriculture ${ }^{2}$. Ainsi, elle cherche à démontrer la réalité cachée derrière le mythe d'un État soviétique qui se serait soucié d'une prise en charge complète des orphelins de guerre, contrairement à ce qui se serait passé dans les autres pays européens. Kucherenko affirme que : «Le bien-être social en temps de guerre continue donc de servir de facteur de légitimation dans la campagne de marketing des régimes visant à prouver la supériorité du socialisme sur le capitalisme » (p. 2).

Kucherenko ne met pas seulement en évidence la discordance entre le mythe et la réalité - ce qui n'est pas étonnant, en raison des conditions de vie de la population à l'époque stalinienne -, elle pointe aussi le fait que l'insertion sociale des orphelins n'était pas une priorité dans un moment de guerre et de combats sur le front. C'est pourquoi le livre « montre comment la politique de l'État a transformé le problème existant en une crise touchant toute la nation, comment l'autoritarisme stalinien a 
étendu les difficultés aux enfants à l'arrière du front et a empêché le gouvernement de répondre efficacement à l'urgence » (p. 3). Cependant, son propos est aussi de montrer les particularités de la politique de l'enfance menée pendant la Seconde Guerre mondiale, c'est-à-dire les mesures de prise en charge et la législation pénale, qui aboutirent à une exclusion sociale des enfants orphelins et délinquants dans un contexte où, après 1943, on assista à un véritable durcissement de cette politique répressive en vue de la fin de la guerre.

Pensait-on que la population allait revendiquer un véritable socialisme après les sacrifices endurés pendant la guerre et cette idée faisait-elle peur ? C'est bien probable. Le régime avait la hantise de perdre le contrôle sur la société, car l'anarchie institutionnelle était un phénomène déjà vu au début des années trente, sous l'impact de la Grande Dépression, dont les effets transnationaux ont été négligés. Mais Kucherenko montre aussi la typologie des institutions organisées pour aborder ce problème d'abandon et de déviance juvénile : la solution pénale n'était pas la seule, bien qu'il y eut une augmentation des internements d'orphelins et de jeunes délinquants dans les DPR et dans les organismes de redressement (comme l'ont aussi montré M.K. Stoole, R. Franco et J. Fürst).

La première partie traite de la bezotcovčina, c'est-à-dire de la perte des parents pendant la guerre et des programmes étatiques vis-à-vis des enfants orphelins et délinquants jetés dans la rue. Du milieu de l'année 1941 jusqu'à la fin de 1945, il y eut environ un million d'enfants abandonnés ou très négligés. Bien qu'il ne s'agisse pas de synonymes du point de vue de la loi, les autorités appliquèrent parfois le même traitement, dévolu aux autorités locales (tout comme cela s'était passé au cours des années vingt avec les besprizornye), avec des attitudes parfois contradictoires. En effet, avant 1943, les seules données disponibles sur leur nombre étaient celles des institutions qui offraient un premier accueil aux enfants, les DPR (detskij priemnik-raspredelitel '), car il n'y avait pas de coordination centrale ni de collecte de données statistiques, devenues impossibles dans le contexte de migration des enfants surtout vers l'Asie centrale. Le lien avec l'absence du père (la bezotcovčina) devint un thème récurrent du discours sur la délinquance juvénile, car effectivement elle avait augmenté de $50 \%$ vers 1944. Il s'agissait de « rolling stones » parcourant le pays, de vagabonds; ni les organisations de jeunesse communiste ni l'école ne pouvaient être une solution. Une population de plusieurs millions de personnes se déplaça vers les républiques de l'est, presque toujours sans aucun support matériel. Les autorités centrales, le Conseil des Commissaires du Peuple (SNK) et le Département de lutte contre l'enfance abandonnée et négligée (OBDBB) du Commissariat du Peuple des Affaires intérieures (NKVD) adoptèrent des mesures concernant la nourriture et la gestion des institutions d'accueil mais, tout comme cela s'était passé dans les années vingt, faute de moyens et dans le contexte de la guerre, le régime fit appel à la responsabilité sociale de la population.

La deuxième et la troisième parties sont complémentaires et offrent une analyse de l'évolution des mesures prises pour placer les jeunes et les réprimer. La deuxième partie reconstitue la façon dont les autorités locales essayèrent de faire face à cette masse de population déplacée, car le gouvernement central et le Parti étaient 
concentrés sur les nécessités du front. Le slogan «Tout pour le front, tout pour la victoire ! » impliqua l'engagement de la population civile face à cette énorme quantité d'enfants (orphelins de père, mais souvent aussi fils des frontoviki [combattants $\mathrm{du}$ front]) lorsque leurs mères s'adressaient aux institutions d'assistance locale. Cette partie traite aussi les questions de rations de nourriture, des déplacements forcés (de l'Est de la Pologne, des États de la Baltique, de la Bessarabie et de la Bucovine du Nord) et celle, très originale, de la transformation « des jeunes travailleurs en criminels » par le biais du service du travail obligatoire, qui entraînait la criminalisation de la violation par les jeunes des normes de travail et une application plus répressive de la justice appliquée aux enfants et aux jeunes. La troisième partie présente la « maison d'État », c'est-à-dire les conditions de vie dans les DPR et les colonies pénitentiaires pour mineurs et les pratiques d'encadrement dans ces institutions pénitentiaires, qui se basaient surtout sur la formation professionnelle par le travail artisanal. Les nombreuses dispositions du NKP et du NKVD visant à l'amélioration de ces institutions, en raison de l'augmentation du nombre d'enfants incarcérés, révèlent une volonté politique de réinsérer les jeunes, bien que sans efficacité. La reconstitution de ce tableau est particulièrement originale. Kucherenko conclut que le régime décida de garder le secret sur ces problèmes sociaux, car c'était une cible possible pour la propagande anticommuniste.

La question de l'imbrication entre prévention et répression est fondamentale pour la compréhension du système soviétique et de son évolution. Les réformes ne furent lancées qu'au milieu des années soixante. On ne peut pas faire grief à l'auteur de ne pas avoir approfondi la notion d'enfant abandonné et délinquant ni la question du durcissement de la répression pénale de l'enfance pour comprendre dans quelle mesure les procureurs de Stalin étaient animés par l'indulgence ou bien par la volonté d'appliquer les lois draconiennes du travail, ces questions ont déjà été abordées par les historiens. On aurait souhaité une définition du welfare de guerre pour décrire les mesures d'intervention vis-à-vis de l'enfance pendant la Seconde Guerre mondiale, compte tenu du fait que le régime avait transformé la protection sociale en outil de contrôle discriminatoire de la productivité des travailleurs dans la période des années trente ${ }^{3}$ et que la protection sociale constitue probablement un des postes les plus onéreux des systèmes totalitaires (par rapport à l'Allemagne par exemple, G. Aly, 2005).

Enfin, ce travail a le mérite de nous offrir de véritables découvertes documentaires : l'État avait la volonté, bien que faible, d'offrir à la population une aide face aux conséquences catastrophiques de la guerre. Parmi les sources originales particulièrement intéressantes, signalons les souvenirs de guerre rassemblés dans l'immédiat après-guerre, tels qu'ils ont été publiés dans un recueil par Nina Petrova sur « la guerre vue par les yeux des enfants », qui constituent des témoignages inédits sur les expériences vécues par les enfants ${ }^{4}$.

Ce livre, qui montre remarquablement comment la guerre a bouleversé la vie des enfants, est servi par une écriture claire. Bien structuré, très agréable à lire et accessible à un large public, il enrichit notre connaissance de cette période dramatique de l'Union soviétique et de l'attitude de l'État face à l'enfance au vingtième siècle, 
offrant une comparaison intéressante avec la réalité de l'Allemagne nazie (N. Stargardt, 2006). Il nous aide aussi à garder la mémoire de la grande contribution de ce pays à la défaite du nazisme.

1 - D. Caroli, L'enfance abandonnée et délinquante dans la Russie soviétique (1917-1937), Préf. de Jutta Scherrer, P. : L'Harmattan, 2004.

2 - J. de Graffenried, Sacrificing Childhood: Children and the Soviet State in the Great Patriotic War, Lawrence : University Press of Kansas, 2014.

3 - D. Caroli, La protection sociale en Union Soviétique (1917-1939). Préf. de Roberto Sani, P.: L'Harmattan, 2010.

4 - N.K. Petrova, Vojna glazami detej : Svidetel'stva očevidcev [La guerre vue par les enfants, témoignages], M. : Veče, 2011 ; N.K. Petrova, « Deti Velikoj Otečestvennoj vojny [Les enfants de la Grande Guerre patriotique] » in A.Ju. Rožkov, éd., Vtoraja mirovaja vojna $v$ detskih "ramkah pamjati": Sbornik naučnyh statej [La Seconde Guerre mondiale dans la mémoire des enfants : Recueil d'articles], Krasnodar : Ekoinvest (Krasnodarskij Gosudarstvennyj Universitet Kul'tury i iskusstv), Rossijskij gumanitarnyj naučnyj Fond, 2010, p. 211-247.

\title{
Dorena Caroli
}

\author{
Université de Macerata - Italie
}

